Publisher homepage: www.universepg.com, ISSN: 2707-4625 (Online) \& 2707-4617 (Print) https://doi.org/10.34104/ijmms.020.051063

International Journal of Material and Mathematical Sciences

Journal homepage: www.universepg.com/journal/ijmms

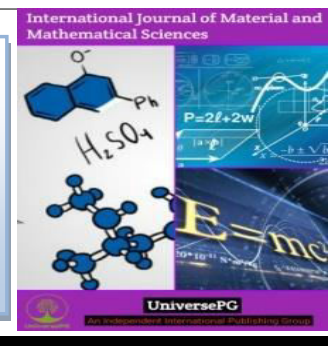

\title{
First Principles Study on the Structural, Elastic, Electronic and Optical Properties of Cubic 'Half-Heusler' Alloy RuVAs Under Pressure
}

\author{
Farhana Mostari ${ }^{1}$, Md. Atikur Rahman ${ }^{2 *}$, Rukayia Khatun ${ }^{3}$ \\ ${ }^{1,2 \& 3}$ Department of Physics, Pabna University of Science and Technology, Pabna-6600, Bangladesh. \\ *Correspondence: atik0707phy@gmail.com (Md. Atikur Rahman, Assistant Professor, Department of Physics, Pabna \\ University of Science and Technology, Pabna, Bangladesh).
}

\begin{abstract}
The pressure effect ( 0 to $40 \mathrm{GPa}$ ) on the structural, elastic, electronic and optical properties of half-metallic compound RuVAs has been investigated employing the DFT based on the first-principles method. The CASTEP computer code is used for this investigation. The calculated lattice parameter show slide deviation from the synthesized and other theoretical data. The normalized lattice parameter and volume are decreased with increasing pressure. The zero pressure elastic constants and also the pressure-dependent elastic constants are positive up to $40 \mathrm{GPa}$ and satisfy the Born stability condition which ensured that the compound $\mathrm{RuVAs}$ is stable in nature. At zero pressure, the electronic band gap of $0.159 \mathrm{eV}$ is observed from the band structure calculations which ensured the semimetallic nature of RuVAs. No band gap is observed in the electronic band structure at $40 \mathrm{GPa}$ which indicates the occurrence of phase transition of compound RuVAs at this pressure. We have calculated the value of bulk modulus $B$, shear modulus $G$, Young's modulus $E$, Pugh ratio $B / G$, Poisson's ratio $v$ and anisotropy factor $A$ of this compound by using the Voigt-Reuss-Hill (VRH) averaging scheme under pressure. The bulk modulus shows a linear response to pressure so that the hardness of this material is increased with increasing pressure. Furthermore the optical properties such as reflectivity, absorptivity, conductivity, dielectric constant, refractive index and loss function of RuVAs were evaluated and discussed under pressure up to $40 \mathrm{GPa}$.
\end{abstract}

Keywords: RuVAs, Crystal structure, Elastic properties, Electronic properties, and Optical properties.

\section{INTRODUCTION:}

Nowadays Half-Heusler alloys are known as the best materials for different applications including optoelectronics, electrical, thermoelectric and piezoelectric applications (Masuri et al., 2016). Ternary form of half-Heusler alloys is $\mathrm{XYZ}$ and of fullHeusler alloys $X_{2}$ YZ (Hülsen et al., 2009; Lee et al., 2005; Galanakis and Mavropoulos, 2007), having four fcc lattices and usually crystallize in $\mathrm{L} 21_{1}$ and $\mathrm{C} 1_{b}$ structure. Where, $\mathrm{X}$ and $\mathrm{Y}$ represents the most and intermediate electropositive element respectively, and $\mathrm{Z}$ denotes the most electronegative element. High spin polarization, half-metallic

UniversePG I www.universepg.com property, and sustain aggressive environment are possessed by Half-Heusler alloys (Andrea et al., 2014; Bhat et al., 2015; Zhang et al., 2014). In the Half-metallic (HM) materials one spin state is metal and the other behaves like semiconducting or insulating. Because of their potential applications making spin electronics devices like spin diode, spin field effect tube, spin throttle valve and spin filter, it plays an important role in materials science (Katsnelson et al., 2008; Li et al., 2004).

The first Heusler compound (NiMnSb) was discovered and observed that there was a gap at the 
Fermi energy for the minority spin channel (usually spin-down), but not for the majority spin channel (usually spin-up). It gives rise to $100 \%$ spinpolarization in one of the spin channels at the Fermi level due to the existence of the band gap. In the half metals, it produces a fully spin-polarized current which helps in maximizing the efficiency of magneto-electronic devices. Like transition metal pnictides and metal-chalcogenides (Ge and Zhang, 2009; and Guo and Liu, 2011), different types of perovskites (Zhu and Yan, 2009), oxides (Hongliang, 2007; Dho et al., 2010), magnetic semiconductors (Kronik et al., 2002; Naefa and Rahman, 2020; Noor et al., 2011), and all types of Heusler alloys (Nour-mohammadi and Abolhasani, 2010; Wang and Wei, 2011) have shown the properties half-metals and this have an important applications in the field of spintronics (Jimbo et al., 1994).

Having outstanding applications and uses in several sectors, Half-Heusler compounds are attracting researchers' attention. Recently, various physical properties are investi-gated of these types of compounds. For example, the electronic and physical properties of $\mathrm{PtBiZ}(\mathrm{Z}=\mathrm{Fe}, \mathrm{Mn}, \mathrm{Ni}, \mathrm{Co})$ are investigated in and of PdMnBi in (Huang et al., 2014). Electronic and magnetic properties of several half-Heusler compounds are investigated and discussed in (Guan-Nan and Ying-Jiu, 2009; Rozale et al., 2013).

Optoelectronic properties of $\mathrm{RbSrZ}(\mathrm{Z}=\mathrm{C}, \mathrm{Si}, \mathrm{Ge})$ are examined in (Ahmad et al., 2013), of CoCrZ (Z $=\mathrm{Al}, \mathrm{Ga}$ ) in (Missoum et al., 2014), and of NiTiX and $\operatorname{CoVX}(\mathrm{X}=\mathrm{Sb}, \mathrm{Sn})$ in (Ameri et al., 2014). Half-Heusler compounds RhCrZ ( $\mathrm{Si}, \mathrm{Ge})$ are investigated in (Mehmood et al., 2017). Though a number of half-Heusler compounds have been predicted to be half-metallic using DFT based calculations (Kandpal et al., 2006), a comprehensive analysis of the structural, mechanical, electronic, and optical properties of the half-Heusler family is necessary, because it is not sure how many halfmetallic half-Heuslers that can be imagined, are stable. Thus a systematic study of the structural stability of the half-Heusler $\left(\mathrm{Cl}_{\mathrm{b}}\right)$ family should provide guidance for future experiments. In this article, we have investigated the structural, electronic, elastic, and optical properties of the half-
Heusler alloys RuVAs at zero and different pressure up to $40 \mathrm{GPa}$. Since only the structural and magnetic properties of compound RuVAs have been studied in (Ma et al., 2017), therefore we are interested to study the detail physical properties of this compound at 0 $40 \mathrm{GPa}$. Here we have tried to show the variation of different physical properties of RuVAs under different pressure.

\section{Computational details}

First-principles calculations have been carried out using the DFT-based CASTEP computer program with the generalized gradient approximation (GGA) and the PBE exchange correlation functional was chosen for this calculation (Clark et al., 2005; Rahman et al., 2016; Perdew et al., 2008). In this calculation V- $3 s^{2} 3 p^{6} 3 d^{3} 4 s^{2}$, As- $4 s^{2} 4 p^{3}, \mathrm{Ru}-4 s^{2}$ $4 p^{6} 4 d^{7} 5 s^{1}$ are used as valence electrons. In a plane-wave basis set the energy cut-off of $400 \mathrm{eV}$ are used for electromagnetic wave function expansion and $12 \times 12 \times 12 \mathrm{k}$-points are used for the sampling of Brillouin zone for all calculations.

In the geometry optimization, the convergence parameters were set as follows: (i) $1.0 \times 10^{-5} \mathrm{eV} /$ atom is set for the total energy difference (ii) $0.001 \AA$ is set for the maximum ionic displacement (iii) 0.03 $\mathrm{eV} / \AA$ is set for the maximum ionic force (iv) 0.05 Gpa is set for the maximum stress. With space group F43m (space gr. No. 216) the Wyckoff positions for $\mathrm{Ru}, \mathrm{V}$ and As are $(0.25,0.25,0.25),(0.50,0.50$, $0.50)$ and $(0,0,0)$ as shown in Fig 1. The bulk modulus can be obtained from the elastic constants and the elastic stiffness constants of RuVAs are obtained using the stress-strain method (Fan et al., 2006)

\section{RESULTS AND DISCUSSION}

\subsection{Structural properties of RuVAs}

The half-Heusler RuVAs compound has cubic structure with the space group F4-3m (216) and the equilibrium lattice parameter has a value of $5.76 \AA$ which is similar to the calculated value. The optimized lattice parameter and the calculated unit cell volume at zero to $40 \mathrm{GPa}$ pressure are tabulated in Table 1. Minimizing total energy the lattice constants and atomic positions have been optimized with a function of normal stress which is shown in Fig 1. 
(a)

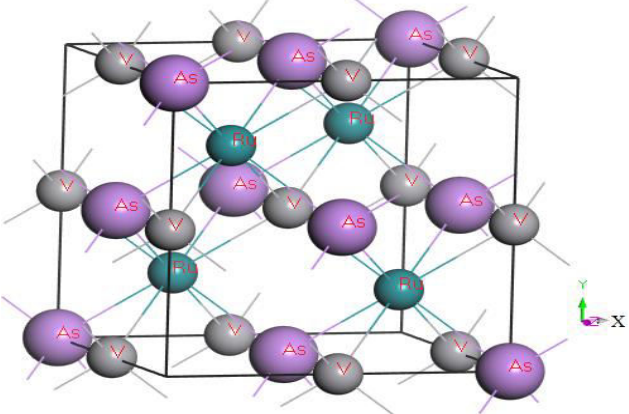

(b)

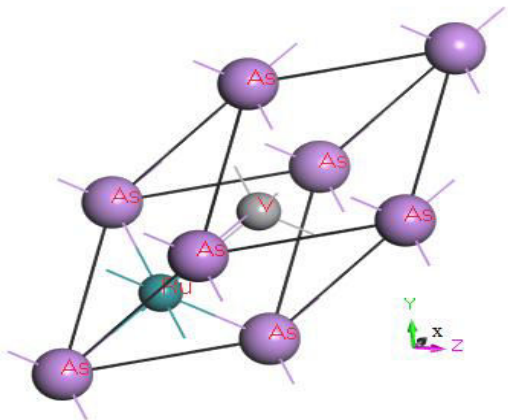

Fig 1: Crystal structure of half-Heusler alloy RuVAs.
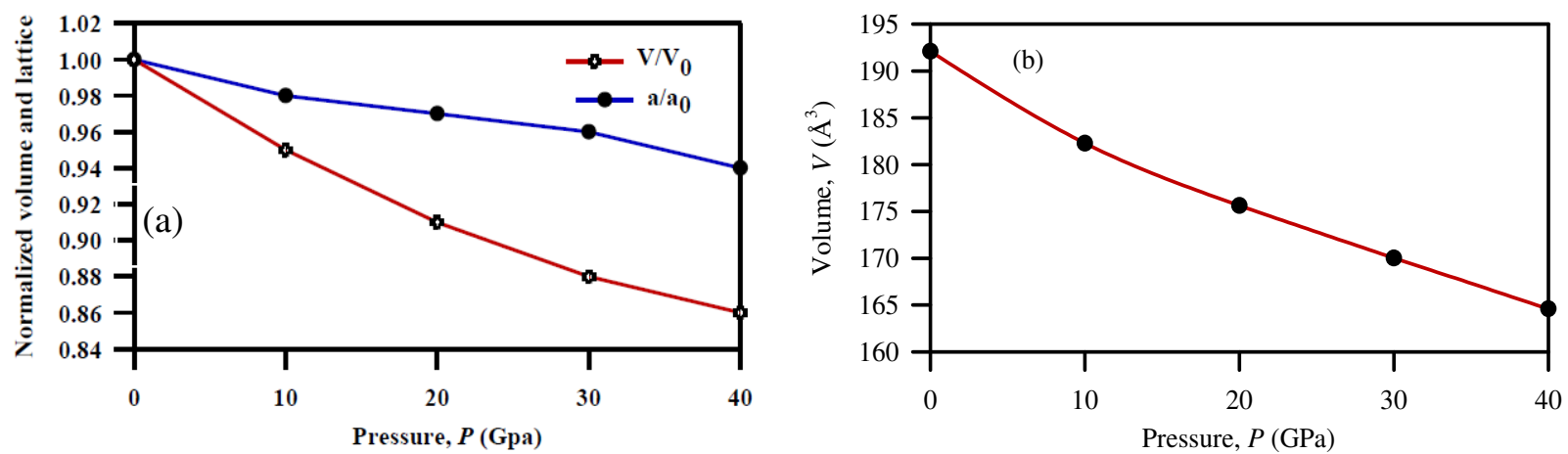

Fig 2: (a) Variations of cell volume and lattice parameter as a function of pressure, and (b) BirchMurnaghan equation of state for RuVAs.

Table 1:The calculated equilibrium lattice parameters $a_{0}(\AA)$, unit cell volume $V_{0}\left(\AA^{3}\right)$, normalized lattice parameter and volume of RuVAs at zero to $40 \mathrm{GPa}$ pressure.

\begin{tabular}{|c|c|c|c|c|}
\hline Pressure & $\begin{array}{c}\text { Lattice } \\
\text { parameter, } \boldsymbol{a}_{\mathbf{0}}(\mathbf{\AA})\end{array}$ & $\begin{array}{c}\text { Volume, } \boldsymbol{V}_{\boldsymbol{o}} \\
\left(\mathbf{\AA}^{\mathbf{3}}\right)\end{array}$ & $\begin{array}{c}\text { Normalized lattice } \\
\text { parameter, } \boldsymbol{a}_{\boldsymbol{~}} \boldsymbol{a}_{\mathbf{0}}\end{array}$ & $\begin{array}{c}\text { Normalized volume, } \\
\boldsymbol{V} / \boldsymbol{V}_{\mathbf{0}}\end{array}$ \\
\hline 0 & $5.77,5.76^{*}[30]$ & 192.10 & 1.00 & 1.00 \\
\hline 10 & 5.67 & 182.28 & 0.98 & 0.95 \\
\hline 20 & 5.60 & 175.62 & 0.97 & 0.91 \\
\hline 30 & 5.54 & 170.03 & 0.96 & 0.89 \\
\hline 40 & 5.48 & 164.57 & 0.95 & 0.84 \\
\hline
\end{tabular}

To study the effect of pressure on the crystal structure of RuVAs, we have studied the variation of lattice parameter and unit cell volume under pressure (0 to $40 \mathrm{Gpa}$ ) which is shown in Fig 2(a). From Fig 2(a), it can be noticed that the ratio $a / a_{0}$ and normalized volume $\mathrm{V} / V_{0}$ decreases with the increase of pressure, where $a_{0}$ and $V_{0}$ represent the equilibrium lattice Parameter and volume at zero pressure, respectively. Due to the reducing of the atomic distance with increasing pressure, the volume decrease. The pressure-volume curves of RuVAs intermetallic from the calculation are shown in Fig 2(b), where the pressure-volume data are fitted to a third order Birch-Murnaghan equation of state (EOS).

\subsection{Elastic properties of RuVAs}

Many different fundamental solid-state properties are closely related with elastic properties of a material (Fu et al., 2008). By the study of elastic constants, the stability of a material, hardness, ductility, brittleness, elasticity, anisotropy nature and other mechanical phenomena are described (Wang et al., 2011). The elastic constants are determined by employing stress strain role belongs to Hook's law. In Table 2 our investigated results of elastic constants are included with available theoretical data. Due to the different calculations method a little bit deviation is observed between the two theoretical values (zero pressure). 
For cubic crystal, the mechanically stability the single elastic constants should convince the Born stability criteria which are following:

$C_{11}>0, C_{44}>0, C_{11}-C_{12}>0$

and $C_{11}+2 C_{12}>0$
From Table 2, we observed that the calculated values of elastic constants are positive and satisfied the above restriction which suggests that the RuVAs is mechanically stable up to $40 \mathrm{GPa}$. There are no other theoretical data available in literature for the elastic constants of RuVAs under pressure and so we are not able to compare our results with other data.

Table 2: Calculated elastic constants $C_{i j}(\mathrm{GPa})$ and Cauchy pressure $\left(C_{12}-C_{44}\right)$ of RuVAs under hydrostatic

\begin{tabular}{|c|c|c|c|c|c|}
\hline Pressure(GPa) & $\boldsymbol{C}_{\mathbf{1 1}}$ & $\boldsymbol{C}_{\mathbf{1 2}}$ & $\boldsymbol{C}_{\mathbf{4 4}}$ & $\boldsymbol{C}_{\mathbf{1 2}}-\boldsymbol{C}_{\mathbf{4 4}}$ & Remarks \\
\hline \multirow{2}{*}{0} & 247.35 & 158.77 & 46.27 & 112.50 & This study \\
\cline { 2 - 5 } & 256.27 & 175.83 & 69.89 & ---- & Chibani et al., 2018 \\
\hline 10 & 300.71 & 202.95 & 70.65 & 132.30 & \multirow{2}{*}{ This study } \\
\hline 20 & 343.45 & 240.17 & 99.89 & 140.28 & \\
\hline 30 & 378.52 & 272.88 & 67.70 & 205.18 & \\
\hline 40 & 416.53 & 309.26 & 85.29 & 223.97 & \\
\hline
\end{tabular}

pressure.
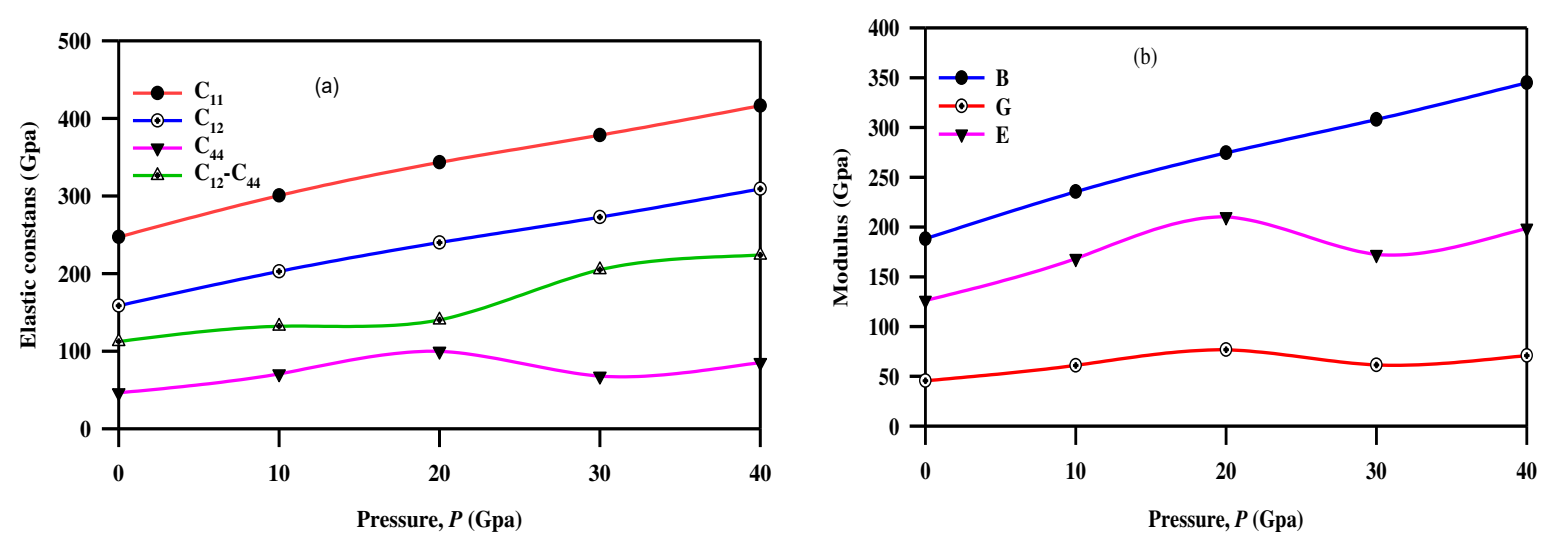

Fig 3: (a) The calculated elastic constants $C_{i j}$ and Cauchy pressure of RuVAs, and (b) Variation of the bulk modulus $B$, shear modulus $G$, Young's modulus $E$ under different pressures.

From Fig 3(a) we observed that the pressure dependence of elastic constants $C_{i j}$ including $C_{11}, C_{12}$ and $C_{44}$ increase with applied pressure except $C_{44}$ because of the lattice parameters become smaller with increasing pressure. The variation of $C_{44}$ which demonstrates that the cubic structure is unstable phase and at high pressure the phase transition may happen. For a cubic crystal, while the elasticity in length is characterized by $C_{11}, C_{12}$ and $C_{44}$ represent the elasticity in shape (Prikhodko et al., 2002). According to Bouhemadou et al. (2015) a transverse strain forms the change in shape with a constant volume where longitudinal strain changes the $C_{11}$.

We can be seen from Table 2 that $C_{11}$ is larger than $C_{44}$ which means that the alloy presents a stronger resistance to the unidirectional than resistance to the pure shear deformation. We have noticed form Fig 3(a) that $C_{11}$ is more sensitive to pressure than $C_{12}$ and $C_{44}$ indicating the shear constant is more sensitive than the Young's modulus. For approximating the brittle or ductile nature of materials an important criterion is Cauchy's pressure, which is given by $\left(C_{12}-C_{44}\right)$. This parameter specified the metallic and nonmetallic feature of material. The positive and negative Cauchy pressure not only prefers the metallic nature and nonmetallic nature respectively (Liu et al., 2012) but also the negative Cauchy pressure indicates the brittle nature and positive Cauchy pressure indicates the ductile nature of material. From our investigation we observed that the Cauchy pressure is positive and increases with increasing pressure confirming the metallic character of RuVAs and this metallic behavior becomes stronger with pressure. For positive value of Cauchy pressure our compound 
shows the ductile manner. For understand the mechanical behavior of materials the polycrystalline elastic parameter such as bulk modulus $B$, shear modulus $G$, Young's modulus $E$ and Poisson's ratio $v$, anisotropy factor $A$ are very vital parameters. Therefore we investigate the variations of these parameters under pressure. We have employed the Voigt-Reuss-Hill (VRH) averaging scheme to estimate these parameters. For cubic crystal, $B$ and $G$ can be expressed as follows (Wu et al., 2007):

$$
\begin{gathered}
B_{v}=B_{R}=\frac{C_{11}+2 C_{12}}{3} \\
G_{v}=\frac{\left(C_{11}-C_{12}+3 C_{44}\right)}{5} \\
G_{R}=\frac{5 C_{44}\left(C_{11}-C_{12}\right)}{\left[4 C_{44}+3\left(C_{11}-C_{12}\right)\right]}
\end{gathered}
$$

The average value of $B$ and $G$ according to Hill can be expressed:

$B=\frac{1}{2}\left(B_{R}+B_{v}\right)$
$G=\frac{1}{2}\left(G_{v}+G_{R}\right)$

The Young's modulus $(E)$ and Poisson's ratio $(v)$ can be determined by using the following relations:

$$
\begin{aligned}
& E=\frac{9 G B}{3 B+G} \\
& v=\frac{3 B-2 G}{2(3 B+G)}
\end{aligned}
$$

The calculated values of $B, G, E$ at different pressures up to $40 \mathrm{GPa}$ are tabulated in Table 3 and plotted in Fig 3(b). The resistance to volume changes and shape changes are measured by bulk modulus, $B$ and shear modulus $G$ respectively. On the other hand, the material stiffness is measured by Young's modulus $E$. The harder of the material is ensured by the large value of Young's modulus. From Fig 3(b) we have observed that with variation of pressure the value of bulk modulus increases which indicate that the stronger the capacity of the resist deformation is increased with increasing pressure. The value of $B$ and $G$ increases with pressure up to $20 \mathrm{Gpa}$ and then decreases. It happens because the anisotropy factor also increases up to $20 \mathrm{GPa}$ and then decreases.

Table 3: The calculated bulk modulus $B$ (GPa), shear modulus $G$ (GPa), Young's modulus $E$ (GPa), $B / G$ values, Poisson's ratio $v$ and anisotropy factor $A$ of RuVAscompound under hydrostatic pressure.

\begin{tabular}{|c|c|c|c|c|c|c|c|}
\hline Pressure(GPa) & $\boldsymbol{B}$ & $\boldsymbol{G}$ & $\boldsymbol{E}$ & $\boldsymbol{A}$ & $\boldsymbol{v}$ & $\boldsymbol{B} / \boldsymbol{G}$ & Remarks \\
\hline \multirow{2}{*}{0} & 188.30 & 45.46 & 126.24 & 1.04 & 0.39 & 4.14 & This study \\
\cline { 1 - 8 } & 202.64 & 55.99 & 153.81 & 1.73 & 0.37 & 3.61 & Chibani et al., 2018 \\
\hline 10 & 235.55 & 60.95 & 168.34 & 1.47 & 0.38 & 3.86 & \multirow{2}{*}{ This study } \\
\hline 20 & 274.60 & 76.65 & 210.37 & 1.93 & 0.37 & 3.58 & \\
\hline 40 & 308.09 & 61.30 & 172.45 & 1.28 & 0.40 & 5.03 & \\
\hline & 345.01 & 70.81 & 198.84 & 1.59 & 0.40 & 4.87 & \\
\hline
\end{tabular}

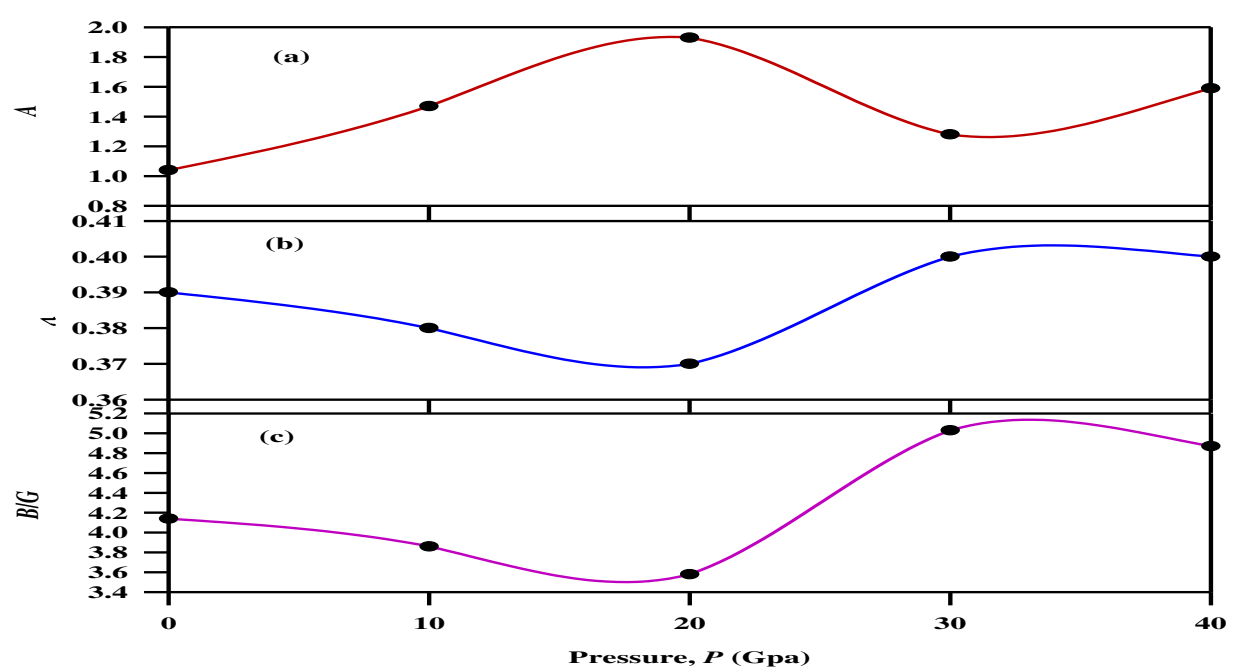

Fig 4: The Anisotropy factor, Poisson's ratio and $B / G$ of RuVAs under pressure. 
The calculated value of $A, v$ and $B / G$ are shown in Fig 4. The Pugh ratio, $B / G$ also provides information about the ductile/brittle nature of any material. If $B / G>1.75$ then the material behaves ductile nature and the material will show brittle nature for $B / G<1.75$. According our results we observed that the values of $B / G$ at different pressure are larger than 1.75 and increases with pressure. So, we can say that with increasing pressure the ductile nature of halfHeusler alloy RuVAs is increases. Poisson's ratio parameter revealed the bonding and stability nature against shear of material and if $v$ ranges between 0.25 and 0.50 (Cao et al., 2013), the inter atomic force in a material is central. The material's flexibility is big with rising of Poisson's ratio. Since the Poisson's ratio are increased with increasing pressure indicating the increase of plasticity with pressure.

The small value of Poisson's ratio for covalent bond is $(v=0.1)$ and for ionic bond $v=0.25$. From Table 3 we see that the value of $v$ are in the range of 0.390.40 , which indicating the existing of central force in RuVAs from 0 to $40 \mathrm{GPa}$. In a solid material the degree of anisotropy is denotes by the Zener anisotropy factor, $A$. For isotropic material ' $A$ ' will be unity whereas values smaller or greater than unity reflect the degree of elastic anisotropy (Evecen, 2017). We have calculated the values of ' $A$ ' utilizing the following expression which are included in Table 3.

$$
A=\frac{2 C_{44}}{\left(C_{11}-C_{12}\right)}
$$

From Fig 4 we can say that the anisotropy behavior of this material is increased up to $20 \mathrm{GPa}$ then it decreases with the increase of pressure.

\subsection{Electronic properties (band structure and density of states)}

To provide valuable data about their experimental modelling and eventual utility in fabricating many type energy devices, the knowledge of the electronic structure is essential (Amudhavalli et al., 2018). To determine the electronic nature of RuVAs the Density of states (DOS) and band structures are calculated within the GGA scheme under pressure up to $40 \mathrm{GPa}$. The band structures in the energy range -8 to $10 \mathrm{eV}$ are seen in Fig 5 and however, energy range remains same with increasing applied pressure. From Fig 5, we can be seen that the conduction band and valance band are not overlapping and the top of the valence band occurs at the $\mathrm{L}$ point and bottom of the conduction band occurs at $\mathrm{X}$ point respectively which implying that the RuVAs compound shows the half-metallic behavior. The band gap of RuVAs compound is $0.159 \mathrm{eV}$ at $0 \mathrm{GPa}$ which is good agreement with the other theoretical values 0.24 (Ma et al., 2017), 0.19 (Shi et al., 2017), 0.09 (Chibani et al., 2018).

From Fig 5, it can be found that without any influence of pressure our compound shows the direct band gap material and it also confirm that after applying external hydrostatic pressure its direct band gap changed into indirect small band gap of $0.073 \mathrm{eV}$ and $0.029 \mathrm{eV}$ at $20 \mathrm{Gpa}$ and $30 \mathrm{Gpa}$ respectively. The band gap almost decreases linearly with the increasing pressure and this trend agrees well with the values obtained by $\mathrm{Wu}$ et al. (2015). The band gap almost decreases linearly with the increasing pressure as shown in Fig 7. It is clear from Fig 5 that when the pressure is applied up to 40GPa the conduction bands shift downwards and valance bands shift upwards and then the band-gap decreases. We can be noticed from Fig 5 there is no band gap at Fermi level at pressure 40 Gpa and then it shows the metallic behavior. So, we say that phase transition occur at $40 \mathrm{GPa}$ of RuVAs compound.

For understanding the bonding the DOS is also an important factor of a material (Duan et al., 2011). The DOS in the energy range -20 to $20 \mathrm{eV}$ for RuVAs under zero pressure are depicted in the Fig 6(a). According to Fig 6 (a) we can see that the major contributions come from As-p and the $d$ states of Ruthenium (Ru) and Vanadium (V). In the case of $\mathrm{Ru}$, at higher energy level the DOS profile is relatively low and flat due to fully occupied d orbits of Ru. Besides, As-p formed the top of the valence band and it keeps at the Fermi level, while the bottom of the conduct band formed by V-d moves downwards, for this reason the band gap decreases. The underlying physical mechanism may be that when the applied pressure increases the distance between As and V shortens and that's why the outer orbitals overlap and then enhances the covalency between As and V, reducing the energy gap between the bonding and anti-bonding orbitals of V-As bonds and ultimately the decrease of the band gap occur. And it is consistent with our investigated band structure. 

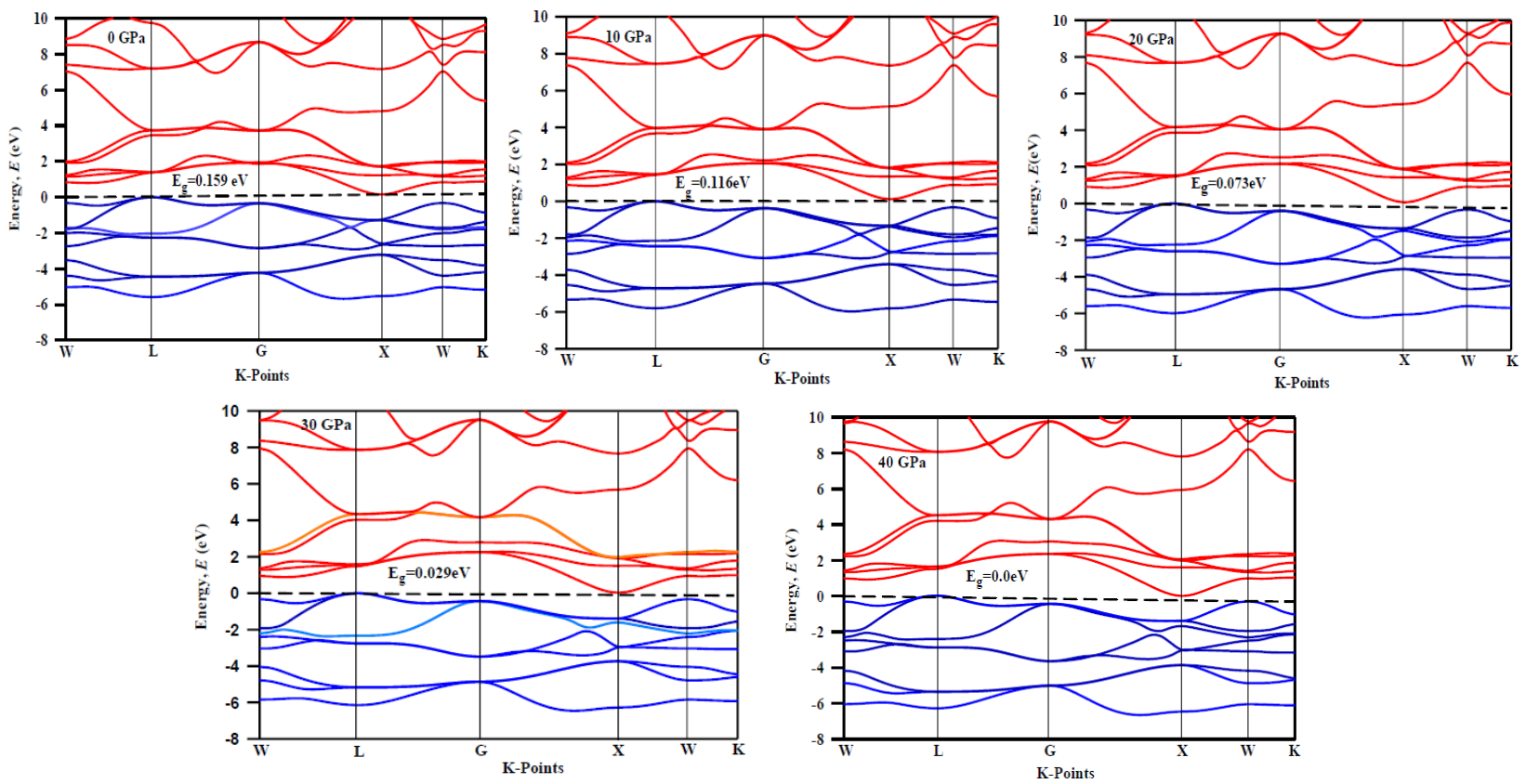

Fig 5: Spin-polarized band structures of the half-Heusler RuVAs.
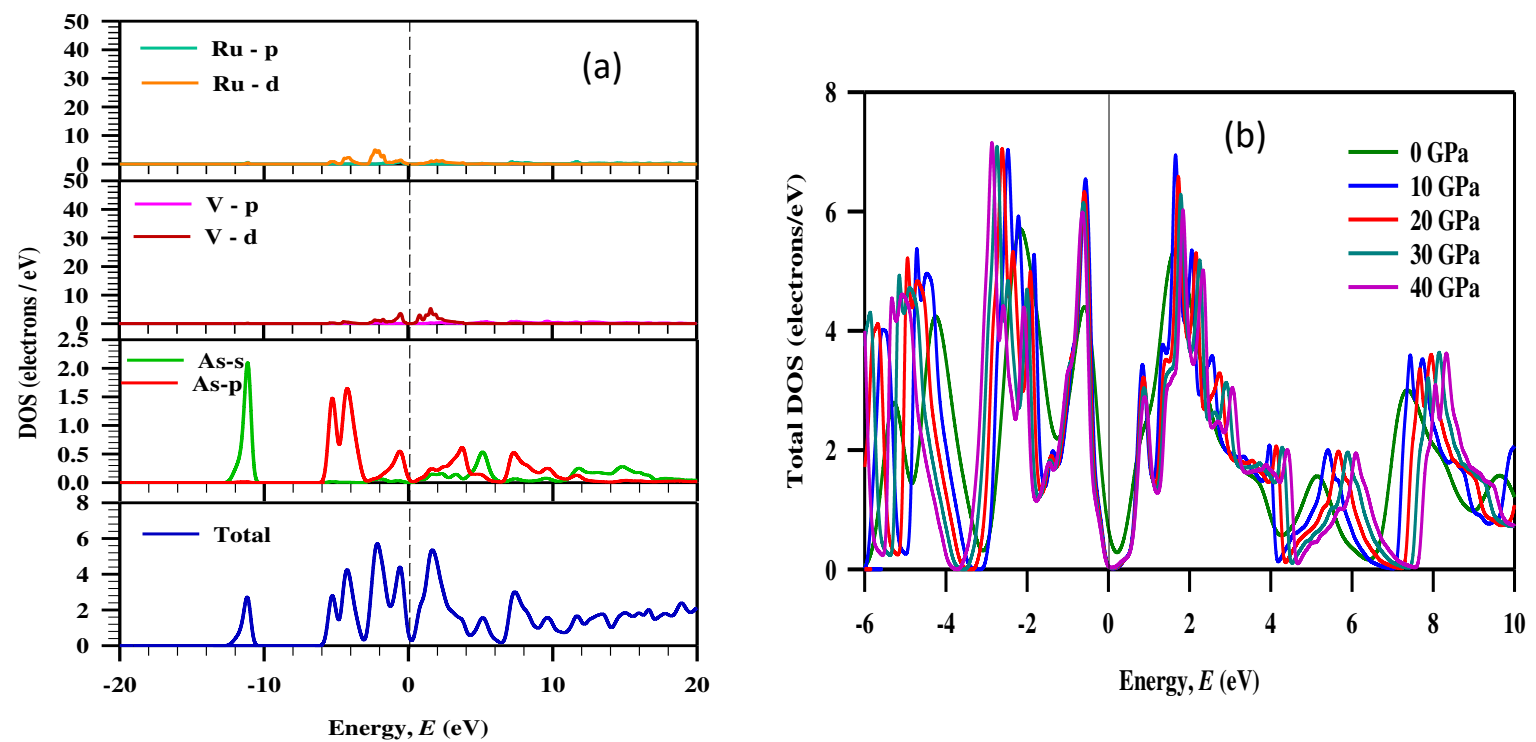

Fig 6: Calculated (a) the partial, and (b) total density of states for RuVAs.

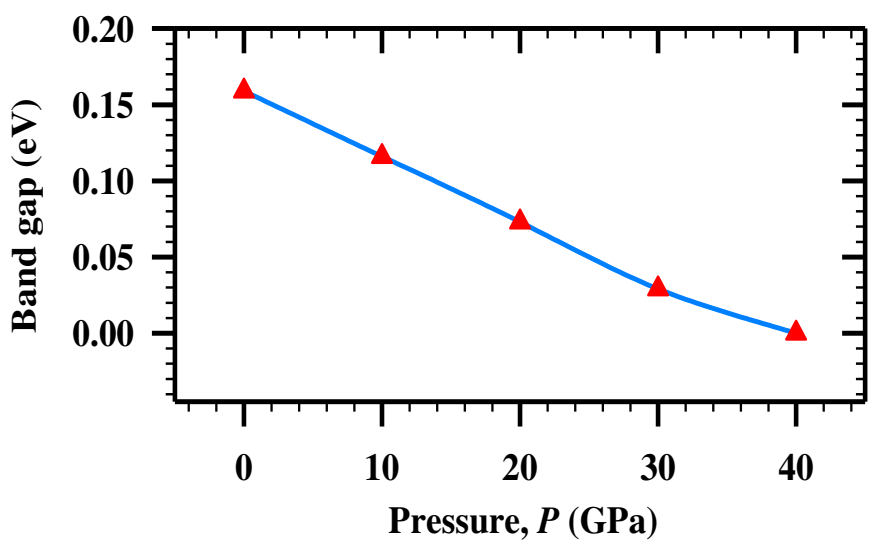

Fig 7: Calculated band gaps of RuVAs under pressure up to $40 \mathrm{GPa}$. 
To elucidate the nature of the Total density of state in the energy range -6 to $10 \mathrm{eV}$ of RuVAs at varying pressures up to $40 \mathrm{GPa}$ were calculated and presented in Fig 6(b). From Fig 6(b) it can be seen that with the increase in external applied pressure, the numbers of new peaks are created and started to appear in the overlap energy states. However, energy range remains same with increasing applied pressure and the lowest energy level almost remain unaffected because of no pressure effect on the lowest energy level. At $0 \mathrm{Gpa}$ the peak is minimum in conduction band, other peak started to seen in conduction band with the rising of pressure (10-40 Gpa). Also, there are lowest energy states in valence band from 0 to 40 Gpa. From Fig 5 we noticed the position of valence band and conduction band almost same at the pressure of $20 \mathrm{Gpa}$ and $30 \mathrm{GPa}$ respectively. At Fermi level the decreases or increases of DOSs correspond to the hardening or softening of the materials, respectively (Yan and Chen, 2014). From our investigation we can be seen that the peak of total DOS at Fermi level are decreasing with increasing pressure which indicate that RuVAs compound become harder with increasing pressure. And it is consistent with the investigation of the elastic properties. Near $E_{\mathrm{F}}$ the main peak is right shifted from valence bands to $E_{\mathrm{F}}$ and this analysis ensured that the delocalization of RuVAs becomes stronger gradually under pressure (Mao et al., 2014).

\subsection{Optical Properties}

In this section, we will discuss the study of optical properties of RuVAs compound under various pressures for understanding the electronic system. For calculating the optical properties of RuVAs, we used the frequency-dependent complex dielectric function which is given by, $\varepsilon(\omega)=\varepsilon_{1}(\omega)+i \varepsilon_{2}(\omega)$ is used.The imaginary part $\varepsilon_{2}(\omega)$ can be written through momentum matrix elements and by considering the transition between the filled and

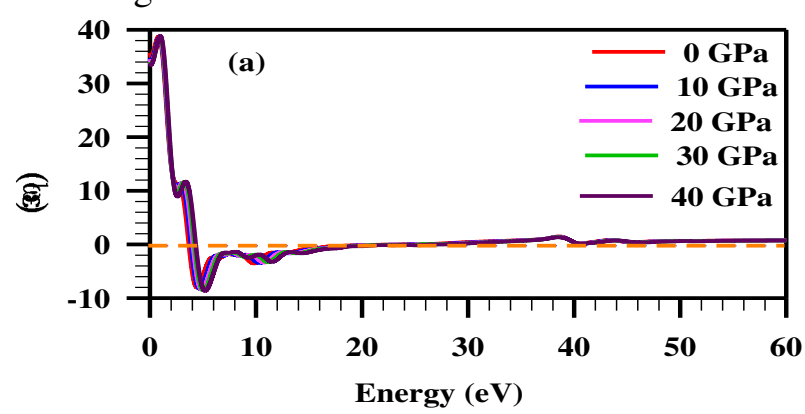

unfilled electronic state by the equation (Accelrys, 2010);

$\varepsilon_{2}(\omega)=\frac{2 \mathrm{e}^{2} \pi}{\Omega \varepsilon_{0}} \sum_{k, v, c}\left|\psi_{k}^{c}\right| u \cdot r\left|\psi_{k}^{v}\right|^{2} \delta\left(E_{k}^{c}-E_{k}^{v}-E\right)$

Where, $u$ is indicating as the polarization vector of the incident electric field, $\omega$ denotes frequency of light, $e$ is denoted as the electronic charge and $\psi_{k}^{c}$ and $\psi_{k}^{v}$ are the conduction and valence band wave functions at $k$, respectively. According to the Kramers-Kronig transform, the real part $\varepsilon_{1}(\omega)$ can be derived from the imaginary part. All other optical constants such as loss-function, refractive index, absorption spectrum, conductivity and reflectivity are calculated using the equations given in (Hadi et al., 2016). For photon energies up to $60 \mathrm{eV}$ the optical functions of these compounds under pressure are calculated. For all calculations the value of Gaussian smearing is $0.5 \mathrm{eV}$.

To understand the polarizability and energy loss of a material, dielectric function is used as a drastic parameter where the electromagnetic wave propagates through it. The real and imaginary dielectric functions at different pressure are shown in Fig 8(a), and (b) respectively. From analysis Fig 8(a), we can see that when with increasing pressure then dielectric constant also increases and from this it can be deduced that flux density also increases for RuVAs. We also noticed that the lower energy limit $\varepsilon_{1}(\omega)$ of dielectric constant is $\varepsilon_{1}(0)$ and peak is gradually disappearing above Fermi level and below the Fermi level getting sharp gradually. For different pressure the value of static dielectric constant changes from 33.66 to 35.66 at 0 to $40 \mathrm{Gpa}$. The imaginary part of $\varepsilon_{2}(\omega)$ shows major peak at visible and minor peak at ultraviolet regions. The absorption and conductivity occurred at the non-zero potion of the imaginary part of the dielectric constant.

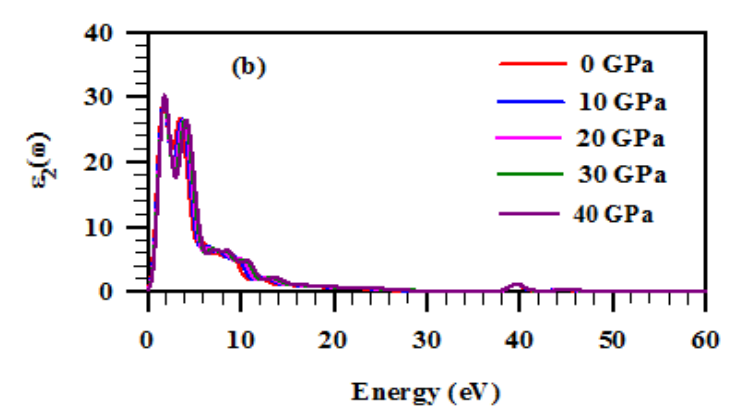

Fig 8: The optical function of pressure dependent (a) real part, and (b) imaginary part of dielectric constant of RuVAs compound. 
The calculated optical reflectivity spectra as a function of photon energy of RuVAs are shown in Fig 9(a). It is observed that the reflectivity is in the range of $0.61-0.63$ in the infrared region for $0-40$ GPa and the value drops rapidly in the visible region (1.8-3.0 eV) and then again increases in ultraviolet region. In our study the highest peaks is between 4.4 to $5.9 \mathrm{eV}$ photon energy ranges of RuVAs compound where the conductivity, loss function fall rapidly and absorption quality is good. To avoid solar heating, the high value of reflectivity in the high energy region can be used as a good coating material. It is so interesting to notice that with the increase of pressure reflectivity also increases. Fig 9(b) shows that the optical absorption co-efficient of RuVAs compound under pressure. The absorption coefficient provides data about the most solar energy conversion efficiency which shows how far of a specific light energy penetrate into the material up to absorption (Roknuzzaman and Islam, 2013).
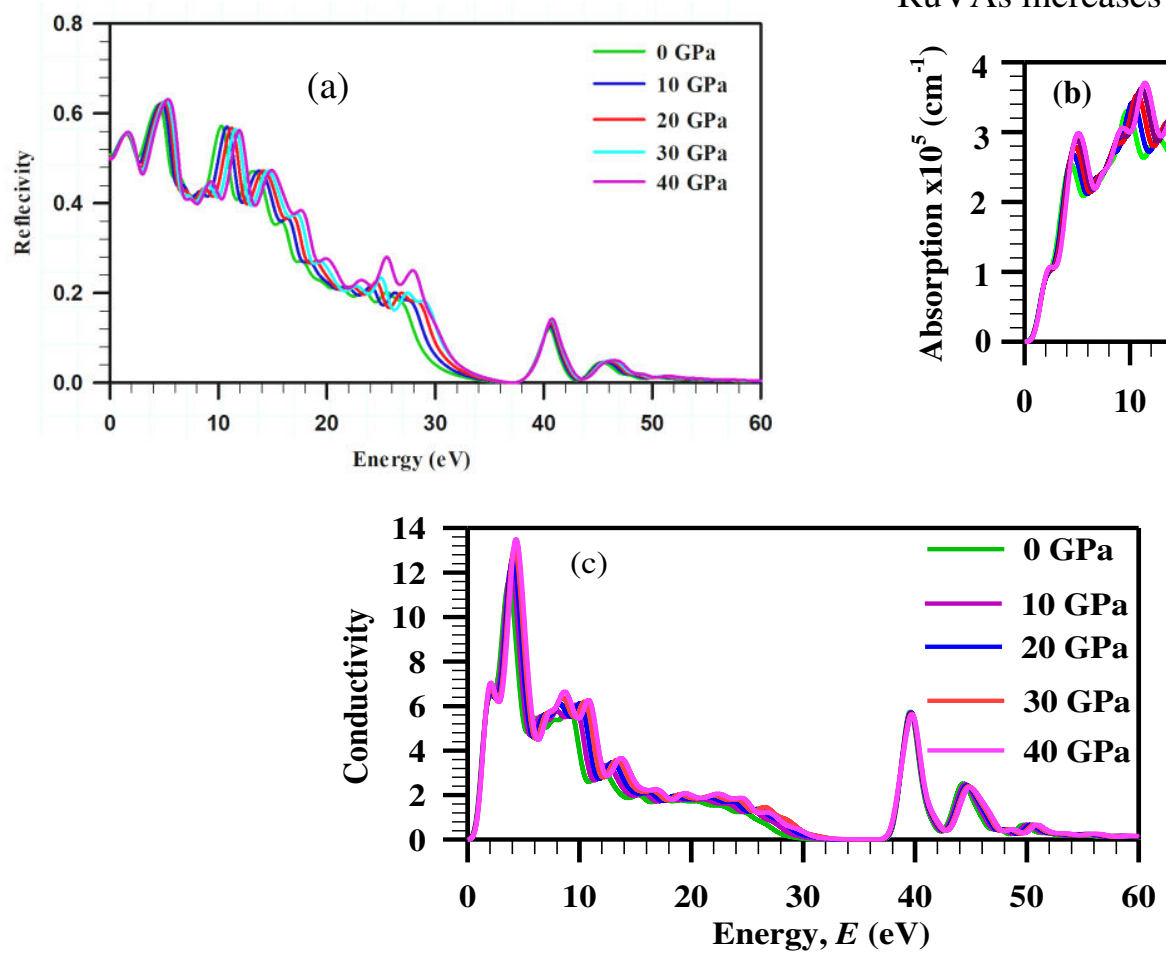

Fig 9: The optical function of pressure dependent (a) reflectivity, (b) absorption, and (c) conductivity of RuVAs compound.

The loss function is a significant parameter and it's explained the energy loss of a fast electron traversing the material (Parvin et al., 2015). The peaks are related to the plasma resonance in the loss function graph and which is associated frequency is called the plasma frequency $\omega_{p}$ and it is shown in Fig 10(a). In the energy range of about $25.5-28.8 \mathrm{eV}$, a few number of large peaks in the energy loss spectra is present. At these high energy range, the real part and UniversePG I www.universepg.com
From $0.2 \mathrm{eV}$ photon energy the absorption coefficient start for RuVAs compound at zero pressure which notifies the small semiconductor behaviors and the corresponding peaks lie at $2.4 \mathrm{eV}, 4.0 \mathrm{eV}$, $4.5 \mathrm{eV}, 6.1 \mathrm{eV}, 6.6 \mathrm{eV}$ from 0-40 GPa. Different peaks are showed in the spectra at the ultraviolet region and there is no absorption from $33.54 \mathrm{eV}$ to $36.96 \mathrm{eV}$ photon energy for (0 - 40) Gpa. At $40 \mathrm{GPa}$ the absorption co-efficient start from zero photon energy because the phase transition occurred in this pressure which ensured from the electronic properties. Due to decrease in band gap the absorption edge changed with respect to pressure. In our study, we notice that RuVAs possess good absorption coefficient in the high energy region and the highest peaks are located at $40 \mathrm{eV}$.

The optical conductivity of RuVAs as a function of photon energy is plotted in Fig 9(c). Due to the result of absorbing photons the photoconductivity of RuVAs increases (Sun et al., 2006).

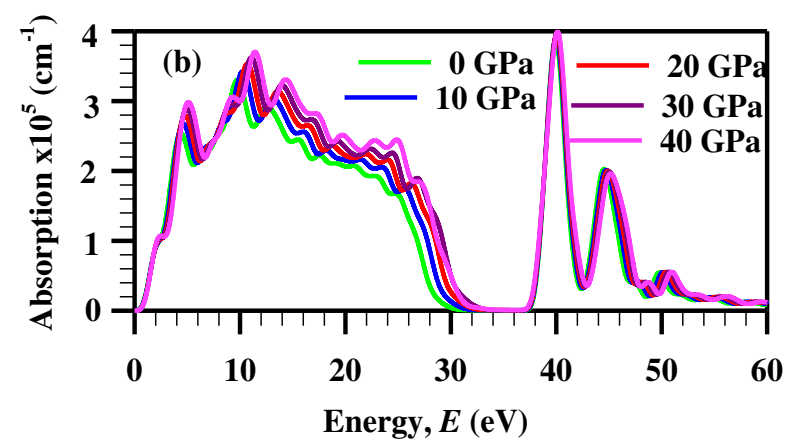

imaginary part of the dielectric function is small and correspond to the trailing edge of $R(\omega)$ (Saniz et al., 2006). With the rising of pressure from 0-30 GPa the loss function is decreasing and with pressure $40 \mathrm{GPa}$ the loss function is increasing because phase transition occurred here. Therefore from energy loss spectrum, the peaks of plasmon energy lie at 26.5 and $28.8 \mathrm{eV}$ respectively, at 0 and $40 \mathrm{GPa}$ pressures, which show that the plasmon energy curve also 
shifts towards the higher energy side with rising pressure. How much light is refracted/bent when entering into material is determined by the refractive index and it is a dimensional quantity (Russell, 2003). The refractive index (real part) at different pressure is shown in Fig 10(b). Within the range up to $40 \mathrm{GPa}$ the refractive index values varies from

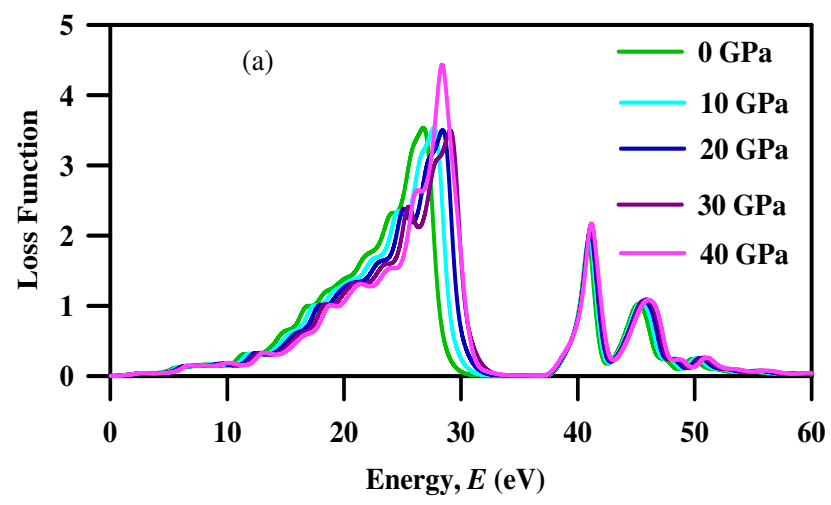

5.81 to 5.95 . From the figure of refractive index we notice that the value of static refractive index is high in the infrared region then highly decrease in the visible region and slowly reduced in the ultraviolet region. The almost similar patterns of the refractive index are observed at various pressures.

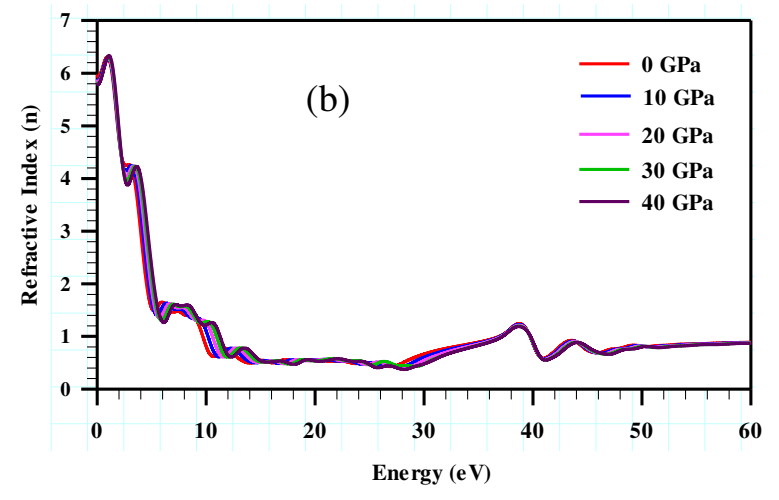

Fig 10: The optical function of pressure dependent (a) loss function, and (b) refractivity index of RuVAs compound.

\section{CONCLUSION:}

In this work, we have investigated the structural, elastic, electronic and optical properties of the halfHeusler alloy RuVAs under various pressures up to $40 \mathrm{GPa}$ by performing first-principles calculations. The structural properties including cell volume and the normalized structural parameters $a / a_{0}$ and $V / V_{0}$ decrease with the increase of pressure. The pressure effects on elastic modulus including bulk modulus $B$, shear modulus $G$ and Young's modulus $E$ show that all the parameters are increases with the increase of pressure. The ductility of these RuVAs compounds have been analyzed using the Pugh's rule and the Cauchy pressure and Pugh's ratio ensured that the compound RuVAs shows ductile behavior. The halfHeusler alloy RuVAs is mechanically stable within the pressure range from $0 \mathrm{GPa}$ to $40 \mathrm{GPa}$ and the material becomes unstable under the pressure more than $40 \mathrm{GPa}$. The compound shows the half-metallic nature with the small indirect band gaps which are revealed from electronic band gap and DOS analysis. The compound RuVAs shows semi-metallic nature up to $30 \mathrm{GPa}$ but it shows totally metallic nature at $40 \mathrm{GPa}$, which ensures the occurring of phase transition of RuVAs compound at $40 \mathrm{GPa}$. The optical properties including dielectric constant, optical absorption, optical conductivity, refractive index, reflectivity and loss function shows the increasing behavior with the increase of pressure. We tried our best to discuss the optical properties of
RuVAs under pressure which might be the key reason to enhance the near/mid infrared light activity.

\section{ACKNOWLEDGEMENT:}

We would like to thank Department of Physics, Pabna University of Science and Technology, Bangladesh for the condensed matter lab support.

\section{CONFLICT OF INTERESTS:}

We have no conflict of interest about this article.

\section{REFERENCES:}

1. Accelrys, (2010). Materials Studio CASTEP manual - Accelrys, (Accelrys, 2010), pp. 261-262.

http://www.tcm.phy.cam.ac.uk/castep/docume ntation/WebHelp/CASTEP.html

2. Ahmad, M., Murtaza, G., and Bouhemadou, A. (2015). Structural, elastic, electronic, magnetic and optical properties of $\mathrm{RbSrX}$ (C, SI, Ge) half-Heusler compounds. J. of Mag. and Magn. Mat., 377, pp.204-210.

3. Ameri, M., Touia, A., Khenata, R., AlDouri, Y. and Baltache, H. (2013). Structural and optoelectronic properties of NiTiX and $\mathrm{CoVX}(\mathrm{X}=\mathrm{Sb}$ and $\mathrm{Sn})$ half-Heusler compounds: An ab initio study. Optik, 124(7), pp.570-574.

https://doi.org/10.1016/j.ijleo.2011.12.052 
4. Amudhavalli, A., Rajeswarapalanichamy, R., Iyakutti, K. and Kushwaha, A.K. (2018). First principles study of structural and optoelectronic properties of $\mathrm{Li}$ based half Heusler alloys. Computational Condensed Matter, 14, pp.55-66.

5. Andrea, L., Hug, G. and Chaput, L. (2014), June. Ab initio lattice thermal conductivity in pure and doped half-Heusler thermoelectric materials, June 2014.

https://hal.archives-ouvertes.fr/hal-01111497

6. Bhat, I.H., Yousuf, S., Bhat, T.M. and Gupta, D.C. (2015). Investigation of electronic structure, magnetic and transport properties of half-metallic $\mathrm{Mn}_{2} \mathrm{CuSi}$ and $\mathrm{Mn}_{2} \mathrm{ZnSi}$ Heusler alloys. J. of Magnetism and Magnetic Materials, 395, pp.81-88.

7. Bouhemadou, A., D. Allali, S. Bin-Omran, and Al-Douri Y. (2015). "Elastic and thermo-dynamic properties of the $\mathrm{SiB}_{2} \mathrm{O}_{4}$ $(\mathrm{B}=\mathrm{Mg}, \mathrm{Zn}$ and $\mathrm{Cd})$ cubic spinels: $\mathrm{An} \mathrm{ab}$ initio FP-LAPW study." Materials Science in Semiconductor Processing, 38: 192-202. https://doi.org/10.1016/j.mssp.2015.04.021

8. Cao, Y., Zhu, J., Liu, Y., Nong, Z. and Lai, Z. (2013). First-principles studies of the structural, elastic, electronic and thermal properties of $\mathrm{Ni}_{3} \mathrm{Si}$. Computational materials science, 69, pp.40-45.

9. Chibani, S., Arbouche, O., Zemouli, M., Benallou, Y., Amara, K., Chami, N., Ameri, M. and El Keurti, M. (2018). First-principles investigation of structural, mechanical, electronic, and thermoelectric properties of HalfHeusler compounds RuVX (X= As, $\mathrm{P}$, and $\mathrm{Sb})$. Computational Condensed Matter, 16, p.e00312.

https://doi.org/10.1016/j.cocom.2018.e00312

10. Clark, S.J., Segall, M.D., Pickard, C.J., and Payne, M.C. (2005). First principles methods using CASTEP. Zeitschrift für Kristallo graphie Crystalline Materials, 220(5-6), pp.567-570.

11. Dho, J., Ki, S., Gubkin, A.F., Park, J.M.S. and Sherstobitova, E.A. (2010). A neutron diffraction study of half-metallic ferromagnet $\mathrm{CrO}_{2}$ nanorods. Solid state communications, 150(1-2), pp.86-90.

https://doi.org/10.1016/j.ssc.2009.09.044
12. Duan, Y.H., Sun, Y., Peng, M.J. and Guo, Z.Z. (2011). First principles investigation of the binary intermetallics in $\mathrm{Pb}-\mathrm{Mg}-\mathrm{Al}$ alloy: stability, elastic properties and electronic structure. Solid State Sciences, 13(2), 455459.

https://doi.org/10.1016/S1003-6326(16)64107-9

13. Evecen, M. (2017). First-principles study on the structural, elastic, electronic and vibrational properties of scandium based intermetalic compounds ( $\mathrm{ScX}, \mathrm{X}=\mathrm{Co}, \mathrm{Rh}$ and $\mathrm{Ir}$ ) under pressure. Journal of Nanoel-ectronics and Optoelectronics, 12(2), 100-108.

14. Fan, C.Z., Zeng, S.Y., Li, L.X., Zhan, Z.J., and Yao, Y.G. (2006). Potential superhard osmium dinitride with fluorite and pyrite structure: First principles calculations. Physical Review B, 74(12), p.125118.

15. Fu, H., Li, D., Peng, F., Gao, T. and Cheng, X. (2008). Ab initio calculations of elastic constants and thermodynamic properties of NiAl under high pressures. Computational Materials Science, 44(2), pp.774-778. https://doi.org/10.1016/j.commatsci.2008.05.026

16. Galanakis, I. and Mavropoulos, P. (2007). Spin-polarization and electronic properties of half-metallic Heusler alloys calculated from first principles. Journal of Physics: Condensed Matter, 19(31), p.315213.

17. Ge, X.F. and Zhang, Y.M. (2009). Firstprinciples study of half-metallic ferromagnetism in $\mathrm{Zn}_{1}-\mathrm{xCrxSe}$. J. of magnetism and magnetic materials, 321(3), pp.198-202.

18. Guo, S.D. and Liu, B.G. (2011). Improved half-metallic ferromagnetism of transitionmetal pnictides and chalcogenides calculated with a modified Becke-Johnson exchange potential. EPL (Europhysics Letters), 93(4), p.47006.

https://doi.org/10.1209/0295-5075/93/47006

19. Guan-Nan, L. and Ying-Jiu, J. (2009). Firstprinciples study on the half-metallicity of half-Heusler alloys: $\mathrm{XYZ}(\mathrm{X}=\mathrm{Mn}, \mathrm{Ni} ; \mathrm{Y}=\mathrm{Cr}$, $\mathrm{Mn} ; \mathrm{Z}=\mathrm{As}, \mathrm{Sb})$. Chinese Physics Letters, 26(10), p.107101.

20. Hadi, M.A., Vovk, R.V. and Chroneos, A. (2016). Physical properties of the recently discovered $\mathrm{Zr}_{2}\left(\mathrm{~A}_{1-1}\right.$-xBix $) \mathrm{C}$ MAX phases. Journal of Materials Science: Materials in Electronics, 27(11), pp.11925-11933. 
21. Hongliang, L. (2007). Fabrication and characterization of nanostructured halfmetals and diluted magnetic semiconductors (Doctoral dissertation). http://scholarbank.nus.edu.sg/handle/10635/16 233

22. Huang, W., Wang, X., Chen, X., Lu, W., and Fong, C.Y. (2014). Structural and electronic properties of half-Heusler alloy Pd-Mn-Bi calculated from first principles. Materials Chemistry and Physics, 148(1-2), pp.32-38. https://doi.org/10.1016/j.matchemphys.2014.0 $\underline{7.005}$

23. Hülsen, B., Scheffler, M. and Kratzer, P. (2009). Thermodynamics of the Heusler alloy $\mathrm{Co}_{2}-\mathrm{x} \mathrm{Mn}_{1}+\mathrm{xSi}$ : A combined density functional theory and cluster expansion study. Physical Review B, 79(9), p.094407.

24. Jimbo, M., Hirano, S., Meguro, K., Tsunashima, S. and Uchiyama, S. (1994). Giant magnetoresistance with low saturation field in $\mathrm{Ni}_{14} \mathrm{Fe}_{13} \mathrm{Co}_{73} / \mathrm{Cu}$ multi layers. Japanese journal of applied physics, 33(6B), p.L850. https://doi.org/10.1557/PROC-313-271

25. Kandpal, H.C., Felser, C. and Seshadri, R. (2006). Covalent bonding and the nature of band gaps in some half-Heusler compounds. Journal of Physics D: Applied Physics, 39(5), p.776.

26. Katsnelson, M.I., Irkhin, V.Y., Chioncel, L., and de Groot, R.A. (2008). Half-metallic ferromagnets: From band structure to manybody effects. Reviews of Modern Physics, 80(2), p.315.

https://doi.org/10.1103/RevModPhys.80.315

27. Kronik, L., Jain, M. and Chelikowsky, J.R. (2002). Electronic structure and spin polarization of $\mathrm{MnxGa}_{1}-\mathrm{xN}$. Physical Review B, 66(4), p.041203. https://doi.org/10.1063/1.1787895

28. Lee, S.C., Lee, T.D., Blaha, P. and Schwarz, K. (2005). Magnetic and half-metallic properties of the full-Heusler alloys $\mathrm{Co}_{2} \mathrm{Ti} \mathrm{X}$ ( $\mathrm{X}=\mathrm{Al}, \mathrm{Ga} ; \mathrm{Si}, \mathrm{Ge}, \mathrm{Sn} ; \mathrm{Sb})$. J. of applied physics, 97(10), p.10C307.

29. Li Q., Gong X., Wang C., Wang J., Ip K. and Hark S. (2004). Adv. Mater. 16:1436.

30. Liu, Y., Hu, W.C., Li, D.J., and Yang, X.J. (2012). First-principles investigation of structural and electronic properties of
$\mathrm{MgCu}_{2}$ Laves phase under pressure. Inter metallics, 31, pp.257-263.

https://doi.org/10.1016/j.intermet.2012.07.017

31. Masuri, N.S., Ahmed, R., Shaari, A., Haq, B.U., and Muhamad, M.N. (2016). First Principles Study of Structural, Electronic and Optical Properties of HALF-HEUSLER Aloys LiMGN, NaMGN and KMGN. Jurnal Teknologi, 78(3).

32. Missoum, A., Seddik, T., Murtaza, G., and Baltache, H. (2014). Ab initio study of the structural and optoelectronic properties of the half-Heusler CoCrZ ( $\mathrm{Z}=\mathrm{Al}, \quad \mathrm{Ga})$. Canadian J. of Physics, 92(10), 1105-1112. https://doi.org/10.1139/cjp-2013-0474

33. Ma, J., Hegde, V.I., Munira, K., Xie, Y., and Butler, W.H. (2017). Computational investigation of half-Heusler compounds for spintronics applications. Physical Review B, 95(2), p.024411.

34. Mao, P., Yu, B., Liu, Z., Wang, F. and Ju, Y. (2014). Mechanical, electronic and thermodynamic properties of $\mathrm{Mg}_{2} \mathrm{Ca}$ Laves phase under high pressure: A first principles calculation. Computational materials science, 88, pp.61-70.

https://doi.org/10.1016/j.commatsci.2014.03.006

35. Mehmood, N., Ahmad, R. and Murtaza, G. (2017). Ab initio investigations of structural, elastic, mechanical, electronic, magnetic, and optical properties of half-Heusler compounds $\mathrm{RhCrZ}(\mathrm{Z}=\mathrm{Si}, \mathrm{Ge})$. J. of Superconductivity and Novel Magnetism, 30(9), pp.2481-2488.

36. Naefa MJ, and Rahman MA. (2020). First principles study of structural, elastic, electronic and optical features of the noncentrosymmetric superconductors SrMGe3 (Where $\mathrm{M}=\mathrm{Ir}, \mathrm{Pt}$, and Pd), Int. J. Mat. Math. Sci., 2(2), 16-28.

https://doi.org/10.34104/ijmms.020.016028

37. Noor, N.A., Ali, S. and Shaukat, A. (2011). First principles study of half-metallic ferromagnetism in Cr-doped Cd-Te. Journal of Physics and Chemistry of Solids, 72(6), pp.836-841.

https://doi.org/10.1016/j.jpcs.2011.04.008

38. Nourmohammadi, A. and Abolhasani, M.R. (2010). First-principle study of full Heusler $\mathrm{CO}_{2} \mathrm{YSi}$ using PBE0 hybrid functional. Solid state communi., 150(33-34), pp.1501-1504. 
39. Perdew, J.P., Ruzsinszky, A., Csonka, G.I., and Burke, K. (2008). Restoring the densitygradient expansion for exchange in solids and surfaces. Physical review letters, 100(13), p.136406. https://doi.org/10.1103/PhysRevLett.100.136406

40. Parvin, F., Hossain, M.A., Ali, M.S. and Islam, A.K.M.A. (2015). Mechanical, electronic, optical, thermodynamic proper-ties and superconductivity of ScGa3. Physica B: Condensed Matter, 457, pp.320-325.

41. Prikhodko, M., Miao, M.S. and Lambrecht, W.R. (2002). Pressure dependence of sound velocities in $3 \mathrm{C}-\mathrm{SiC}$ and their relation to the high pressure phase transition. Physical Review B, 66(12), p.125201. https://doi.org/10.1103/PhysRevB.66.125201

42. Rahman, M.A., Rahaman, M.Z. and Rahman, M.A. (2016). The structural, elastic, electronic and optical properties of $\mathrm{MgCu}$ under pressure: A first-principles study. International J. of Modern Physics $B, 30$ (27), p.1650199.

43. Roknuzzaman, M. and Islam, A.K.M.A. (2013). Ab Initio Investigation of Nitride in Comparison with Carbide Phase of Superconducting $\mathrm{InX}(\mathrm{X}=\mathrm{C}, \mathrm{N})$. International Scholarly Research Notices, 2013. https://doi.org/10.1155/2013/646042

44. Rozale, H., Amar, A., Lakdja, A., Moukadem, A. and Chahed, A. (2013). Halfmetallicity in the half-Heusler $\mathrm{RbSrC}$, $\mathrm{RbSrSi}$ and $\mathrm{RbSrGe}$ compounds. $J$. of magnetism and magnetic materials, 336, 8387. https://doi.org/10.1016/j.jmmm.2013.02.024

45. Russell, P., (2003). Photonic crystal fibers. science, 299(5605), pp.358-362.

46. Saniz, R., Ye, L.H., Shishidou, T. and Freeman, A.J. (2006). Structural, electronic, and optical properties of $\mathrm{NiAl}_{3}$ : firstprinciples calculations. Physical Review B, 74(1), p.014209.
47. Shi, F., Si, M.S., Xie, J., Mi, K., and Luo, Q. (2017). Hybrid density functional study of bandgaps for 27 new proposed half-Heusler semiconductors. Journal of Applied Physics, 122(21), p.215701. https://doi.org/10.1063/1.4998145

48. Sun, J., Zhou, X.F., Fan, Y.X., Chen, J., and Tian, Y., (2006). First-principles study of electronic structure and optical properties of hetero diamond $\mathrm{BC}_{2} \mathrm{~N}$. Physical Review B, 73(4), p.045108.

49. Wang, W.Z. and Wei, X.P. (2011). Halfmetallic anti ferromagnetic in $\mathrm{Mn}_{2} \mathrm{ZnCa}$. Computational materials science, 50(7), pp.2253-2256.

50. Wu, Z.J., Zhao, E.J., Xiang, H.P., Hao, X.F., Liu, X.J. and Meng, J. (2007). Crystal structures and elastic properties of superhard $\mathrm{IrN}_{2}$ and $\mathrm{IrN}_{3}$ from first principles. Physical Review B, 76(5), p.054115.

https://doi.org/10.1103/PhysRevB.76.054115

51. Wu, H.Y., Chen, Y.H., Deng, C.R., Han, X.Y. and Liu, Z.J. (2015). Electronic, elastic and dynamic properties of the filled tetrahedral semiconductor LiMgN under pressures. J. of Solid State Chemistry, 231, 1-6.

52. Yan, M.F. and Chen, H.T. (2014). Structural, elastic and electronic properties of $\mathrm{Cr}_{2} \mathrm{~N}$ : A first principles study. Computational materials science, 88, pp.81-85. https://doi.org/10.1016/j.commatsci.2014.02.035

53. Zhang, Y.J., Liu, Z.H., and Liu, G.D. (2014). Magnetism, band gap and stability of halfmetallic property for the quaternary Heusler alloys CoFeTiZ (Z= Si, Ge, Sn). Journal of alloys and compounds, 616, pp.449-453. https://doi.org/10.1016/j.jallcom.2014.07.165

54. Zhu, Z.H. and Yan, X.H. (2009). Halfmetallic properties of perovskite $\mathrm{BaCrO}_{3}$ and $\mathrm{BaCr}_{0.5} \mathrm{Ti}_{0.5} \mathrm{O}_{3}$ super lattice: LSDA+U calculations. J. of Applied Physics, 106(2), p.023713. https://doi.org/10.1063/1.3182721

Citation: Mostari F, Rahman MA, and Khatun R. (2020). First principles study on the structural, elastic, electronic and optical properties of cubic 'half-Heusler' alloy RuVAs under pressure, Int. J. Mat. Math. Sci., 2(4), 51-63. https://doi.org/10.34104/ijmms.020.051063 क्) 